\title{
LA CONCENTRACIÓN EMPRESARIAL Y ESTRATEGIAS DE INCLUSIÓN
}

\author{
Fernando Córdova León, Gabriela Duque Espinoza \\ y Adrián Sigüencia Muñoz ${ }^{a}$
}

Fecha de recepción: 17 de junio de 2020. Fecha de aceptación: 27 de octubre de 2020.

https://doi.org/10.22201/iiec.20078951e.2021.205.69708

Resumen. El crecimiento económico inclusivo y sostenible, objetivo fundamental de desarrollo, busca evitar la desigualdad. Bajo este contexto, se tiene como objeto determinar las características de las empresas con mayor concentración de riqueza en Ecuador, así como identificar las variables que inciden en la inclusión empresarial, y proponer alternativas para un crecimiento inclusivo con participación de MIPyME. Mediante el coeficiente Gini se establece la concentración económica y se estudia la gestión financiera de las empresas. Los resultados revelaron altos índices de desigualdad, rentabilidad sobre activos, endeudamiento, edad y apalancamiento financiero, como predictores de la probabilidad de formar parte del grupo de éxito empresarial. Se evidenció así, la necesidad de implementar políticas públicas orientadas al fomento de la productividad y de las acciones de responsabilidad social.

Palabras clave: Ecuador; crecimiento económico; empresas; gestión financiera; concentración de la riqueza.

Clasificación JEL: D25; M11; M13; N16; N36; O12.

\section{BUSINESS CONCENTRATION AND INCLUSION STRATEGIES}

\begin{abstract}
Inclusive and sustainable economic growth is a fundamental objective of development, and one which seeks to address inequality. Working from this premise, this article sets out to determine the characteristics of the companies with the highest wealth concentration in Ecuador, as well as to identify the variables that affect business inclusion, and to propose alternatives for inclusive growth with the participation of MSMEs. This article employs the Gini coefficient to determine the level of economic concentration and to study companies' financial management models. The findings revealed high inequality indexes: profitability over assets, indebtedness, age, and financial leverage as predictors of the probability of belonging to the most successful category. There is therefore a clear need to implement public policies aimed at promoting productivity and socially responsible behavior.
\end{abstract}

Key Words: Ecuador; economic growth; firms; financial management; wealth concentration.

\footnotetext{
${ }^{a}$ Universidad del Azuay, Ecuador. Correos electrónicos: jfcordova@uazuay.edu.ec, gduque@uazuay. edu.ecy asiguencia@uazuay.edu.ec, respectivamente.
} 


\section{INTRODUCCIÓN}

La riqueza puede ser definida como el total de bienes, servicios, trabajo y recursos naturales que posee una economía. Su distribución ha sido un tema controversial debido a la formación de grupos económicos que poseen alta influencia en la toma de decisiones de los gobiernos.

Por otro lado, la equidad social puede entenderse desde diferentes dimensiones como la justicia social, la igualdad de oportunidades, la igualdad de derechos, las prestaciones públicas equitativas, la adecuada distribución de la riqueza, entre otras; todas deben ser abordadas, a su vez, desde diferentes perspectivas, ya que la desigualdad está presente en todas ellas.

Según Calvo et al. (2016), las instituciones económicas y políticas, y la base legal proveen el contexto en el cual funcionan los mercados, estableciendo incentivos y restricciones para los diversos actores. Por su parte, el Banco Mundial (2000) asevera que las políticas tendientes a la búsqueda de equidad se traducen en políticas fiscales, económicas, laborales y sociales, en las que los gobiernos y las corporaciones locales tienen que hacer frente a la pobreza y a la exclusión; por ello, desde lo local, mediante políticas gubernamentales o empresariales se puede contribuir a una sociedad más justa y equitativa.

La desigualdad puede evidenciarse en cifras mundiales. Por ejemplo, el 1\% más rico de América Latina y el Caribe concentra 37\% de la riqueza, mientras el $50 \%$ más pobre sólo accede al 3.5\% de la riqueza total de la región (Oxford Committee for Famine Relief, 2018). Esta inequidad puede disminuir con la introducción de estrategias que permitan mayor igualdad sobre la distribución del ingreso, lo que se conoce como inclusión.

Según la Organización de las Naciones Unidas para la Educación, la Ciencia y la Cultura (UNESCO), se puede definir a la inclusión como un proceso que garantiza que toda persona sea "parte de" y que no permanezca "separado de" (Organización de las Naciones Unidas para la Educación, la Ciencia y la Cultura [unesco], 1989); lo que quiere decir que los sistemas establecidos proveerán acceso y participación recíproca en cualquier ámbito.

A pesar del crecimiento económico agregado mundial, existen países y regiones, como América Latina y el Caribe, cuyo crecimiento económico, productividad laboral y generación de fuentes de empleo decente se han desacelerado, lo que disminuye significativamente el nivel de vida y los salarios de sus habitantes. Además de haberse revertido la tendencia de reducir la economía informal, los datos para 2015 y 2016 muestran un aumento en el empleo informal no agrícola que afecta a casi $47 \%$ de la fuerza laboral de la región (Organización Internacional del Trabajo [отт], 2017). 
Según Apetrei et al. (2019), la inclusión puede ser abordada no sólo desde el aspecto económico; sino también, desde una perspectiva social y laboral. Poner atención en estos ámbitos permite que la productividad de un país en general se incremente, consiguiendo un crecimiento económico y aumento de fuentes de empleo. Los determinantes en la desigualdad y el arraigo de la pobreza en economías en vías de desarrollo incluyen: deficiencias en la formación de capital humano, falta de inversión, reducidas tasas de crecimiento del Producto Interno Bruto (РIB) y poca apertura comercial (Nguyen et al., 2020).

Al hablar de la discrepancia en el reparto de la riqueza, es importante reflexionar sobre la brecha existente entre un elevado nivel de pobreza y una importante acumulación de riqueza perteneciente a pocos; además, de la exclusión de sectores que no generan un fuerte valor agregado o que poseen un bajo nivel de desarrollo, ya sea por razones tecnológicas, de mercado o incluso políticas. América Latina es una región que muestra esta desigualdad en la distribución de la riqueza.

El nivel de concentración de una economía puede ser cuantificada y el índice de Gini es una valiosa herramienta que, al igual que otras, permite medir este comportamiento. En Ecuador este fenómeno, dada su importancia, ha sido objeto de estudio; en el 2000 la desigualdad en Ecuador poseía un coeficiente de Gini del 0.76 (Solimano, 2015). De la misma manera, un estudio más reciente revela que el índice de Gini es de 0.68 (Deere y Contreras, 2011), demostrando que, a pesar de los avances tecnológicos y la globalización, la riqueza permanece concentrada en un bajo porcentaje de la población.

En el contexto económico, las organizaciones existen no sólo con una finalidad de producción o servicio, sino fundamentalmente son actores sociales en el que se involucran recursos financieros, materiales, humanos y tecnológicos; por tanto, las empresas coadyuvan a una distribución equitativa de la riqueza, acceso al empleo, oportunidades, capacitación, y desarrollo personal y profesional de los ciudadanos que componen la sociedad, apoyando a la construcción de un verdadero balance intercultural y social.

Así, una unidad empresarial debe ser también entendida como una comunidad, en donde se busca el equilibrio en todos sus actores, afrontando problemas de exclusión, desempleo, y discriminación, al generar trabajo justo, equitativo, dotando de fuentes de ingreso, y brindando oportunidades y acceso al desarrollo de competencias, lo que aporta a la igualdad y a un mejor vivir; por lo que, la equidad empresarial contribuye a una mayor equidad social.

Las empresas, como sujetos activos de un país, inciden en diversos procesos económicos, políticos o culturales, en la sociedad en la cual se desarrollan; convirtiéndose así en parte de los gestores principales que promueven la inclusión 
en búsqueda de un balance social. Un análisis del comportamiento económico del sector empresarial determinará la existencia de concentración de ingreso, riqueza y poder que atenta contra las posibilidades de desarrollo de un país.

El presente estudio se enfoca en el sector empresarial, ya que es éste el encargado de generar productos y servicios, oportunidades y empleo, lo que se ve reflejado en los ingresos percibidos por estas unidades económicas, creando así la necesidad de desarrollar estrategias que permitan a la empresa una adecuada inserción e inclusión dentro del sector en el cual se desarrolla.

La inclusión económica combina áreas macro y microeconómicas que pueden ser desarrolladas en búsqueda de la innovación empresarial necesaria para alcanzar una nueva distribución de la riqueza, disminuyendo así el coeficiente de Gini.

Este estudio pretende determinar las características de las empresas que presentan el mayor nivel de concentración de riqueza; lo que posibilita a su vez identificar las principales variables que inciden en la inclusión de una compañía en el grupo de mayor concentración, y propone alternativas encaminadas a un crecimiento inclusivo en el que participen activamente las micro, pequeñas y medianas empresas (MIPyME).

Asimismo, la presente investigación abarca diversos aspectos a tratar. El análisis de la concentración económica de los sectores industriales muestra una realidad respaldada con datos empíricos que da indicios sobre la poca competitividad existente en la economía de un país en vías de desarrollo; así como el poder de ciertos grupos económicos para establecer barreras que impiden el desarrollo competitivo de las MIPyME. Los resultados serán útiles para focalizar planes y políticas públicas destinadas a reducir la concentración económica y fomentar la equidad social, mediante la participación equitativa de los agentes sociales en las actividades económicas. La aplicación del índice de Gini al sector empresarial permite medir los focos de desigualdad, en búsqueda de la concienciación hacia la equidad, mediante la reflexión de la desigualdad y el accionar empresarial sobre su quehacer financiero para propender a una sociedad más justa y equitativa.

El artículo se compone de una primera sección como introducción en donde se recalca la importancia del debate sobre distribución y generación de la riqueza en una economía; la segunda sección abarca la literatura relacionada con el objeto del estudio; mientras que la tercera describe los datos y la metodología empleada de la que se obtienen los resultados, mismos que serán analizados y discutidos en la cuarta sección. La sección final presenta las conclusiones de la investigación. 


\section{REVISIÓN DE LA LITERATURA}

El desarrollo sostenible es definido como el resultado de un equilibrio de factores como crecimiento económico, cuidado medioambiental y equidad social; sin embargo, este último concepto es habitualmente omitido en la práctica del desarrollo (Trudeau, 2018). No existe un consenso respecto a cómo medir la equidad social, ya que puede ser abordada desde diversos enfoques como el económico, salud, transporte, urbanismo, género, entre otros (Ballet et al., 2020).

En lo concerniente al presente trabajo, se aborda la equidad social desde el punto de vista económico y empresarial, conceptualizada como la capacidad de los agentes para participar equitativamente de las actividades económicas y la renta generada por éstas (Ncube et al., 2019).

En cuanto a las actividades empresariales, la equidad social puede ser analizada de formas alternativas. En la literatura reciente las más usuales corresponden a la evaluación de la responsabilidad social corporativa y las acciones de stake holders; sin embargo, estos enfoques no abordan los problemas de raíz de la equidad social en lo que respecta a la sostenibilidad. Al tratar la equidad social a nivel de empresa, en términos de desigualdad de ingresos, se puede observar específicamente la contribución de las organizaciones a la igualdad de ingresos del sistema general y, por lo tanto, a la equidad social. Este enfoque da mayor claridad a los tipos de políticas que las empresas y los gobiernos deben implementar para gestionar y mejorar los aspectos sociales (Husted, 2011).

En un entorno económico competitivo, la reducción del desequilibrio en los ingresos empresariales influye positivamente en la equidad social a través de la generación del crecimiento económico y al valor ańadido, lo que permite repartir una mayor cantidad de ingresos entre los diferentes actores sociales. Todo lo anterior deriva en una mayor generación de fuentes de empleo formal con mejores condiciones salariales, gracias al crecimiento de la demanda laboral, y a una mayor y más diversa oferta de bienes y servicios a disposición de consumidores, a la que probablemente no podrían acceder en condiciones de un mercado con alta concentración empresarial, aspectos que permiten que los beneficios del crecimiento económico lleguen a todas las personas (Carneiro et al., 2015). Ncube et al. (2019) presentan evidencia empírica que relaciona la participación de MIPyME en la percepción de ingresos de forma equitativa con un mayor crecimiento económico, mayor participación femenina en el trabajo formal y menor mortalidad infantil. 
Una manera de medir la distribución y concentración de la riqueza es la utilización de los ingresos o beneficios generados por las organizaciones. Esto causa una discusión para el análisis de los sectores económicos y su equilibrio, pues se ha determinado que existe una disparidad en esta distribución y que puede ser abordada desde la "inclusión", lo que implica introducir, o incrementar la participación de los sectores menos representativos dentro de una economía. Según Infante y Sunkel (2009), esta herramienta debería implementarse a largo plazo con el fin de generar un desarrollo inclusivo e imparcial para evitar la desigualdad de los ingresos y mejorar la calidad de vida.

Existe una disparidad en la disponibilidad de los recursos económicos entre individuos, lo que genera una desigualdad social y económica que da como resultado el incremento de la brecha entre individuos ricos y pobres, así como de sus oportunidades (Oxford Committee for Famine Relief, 2018). Entonces, para una correcta inclusión económica se debe contar con apoyo, tanto del sector público como del sector privado, mediante una colaboración que permita determinar distintas herramientas encaminadas a generar una inclusión de empresas y sectores, fomentando así el crecimiento económico y la distribución equitativa del ingreso en un país.

En primera instancia se encuentra el ámbito empresarial, en donde la desigualdad incluye a un número relativamente bajo de participantes que controlan el mercado y la dirección de la actividad económica; lo cual genera, en el peor de los casos, una situación de monopolio; sin embargo, la misma iniciativa empresarial puede contribuir a mejorar el crecimiento económico y la prosperidad cuando se reduce la desigualdad y la exclusión, así se valoran las relaciones entre nuevas empresas y la reducción de las desigualdades a través de mecanismos en los cuales los procesos empresariales contribuyen a las transformaciones productivas y sociales (Apetrei et al., 2019).

Abosede y Onakoya (2013) determinan que el proceso de creación de nuevas empresas introduce y desarrolla distintos productos innovadores, procesos y estructuras organizativas en toda la economía; es decir, fomenta la inclusión económica y reduce las estructuras de acumulación de poder de mercado y riqueza como en el monopolio.

La necesidad de un crecimiento económico inclusivo no se dirige sólo a los países en vías de desarrollo; por ejemplo, en el plan para Europa 2020, el emprendimiento inclusivo estima incluir $75 \%$ de la población entre 20 y 64 años. Este emprendimiento plantea incorporar a parte de la población económicamente activa que no se encuentra trabajando, pero que desea hacerlo, en el desarrollo de nuevas empresas dentro de sectores que aporten en un futuro a la economía y al crecimiento del país. El emprendimiento debe ser innovador, 
pudiendo desarrollarse en cinco categorías: introducción de un nuevo bien o mejorar la calidad de un bien ya existente; nuevo método de producción; apertura de un nuevo mercado; explotación de una nueva fuente de suministro de materias primas; y realización de una nueva organización de cualquier industria (Abosede y Onakoya, 2013).

Los mercados actuales atraviesan cambios constantes, en donde aspectos como la internacionalización de la empresa, ventajas comparativas en comercio exterior, competitividad, creación e innovación, y globalización determinan la competitividad de las economías y la sostenibilidad de las empresas (Valbuena y Montenegro, 2019). En este contexto, las MIPyME que no se adaptan favorable y oportunamente a estos cambios ven reducida su competitividad, de tal suerte que la riqueza generada se concentra en unas cuantas manos, ya que las grandes empresas establecen las condiciones de las industrias, quedándose con la mayoría de los beneficios generados (Oxford Committee for Famine Relief, 2018).

Por otro lado, se encuentra el ámbito macroeconómico y la política económica; es decir, la participación del sector público para solventar la redistribución del ingreso. Un estudio realizado en Chile en 2009 determinó que se necesita un cambio en el enfoque de las políticas públicas para así poder superar paso a paso la diferencia estructural de los sectores de la matriz productiva, obstáculo principal para lograr un crecimiento imparcial, desarrollando cuatro elementos: convergencia productiva, protección social garantizada, cambios institucionales necesarios y crecimiento económico sostenido con equidad (Infante y Sunkel, 2009).

Existen numerosos autores que se apoyan en el índice de Gini y la curva de Lorenz para determinar desigualdad y distribución del ingreso, como es el caso de Alonso (2010) quien descompone a la curva de Lorenz en subsectores con respecto a la industria manufacturera en España. Mientras que Zhao et al. (2007) analizan las disparidades de la misma industria en el caso de China utilizando el índice de Gini; Arbeletche y Carballo (2008) lo estudian en una investigación sobre la concentración agrícola uruguaya. Dávila (2004) aplica una variante del índice de Gini y la curva de Lorenz para medir la evolución de la concentración del empleo manufacturero en México; mientras que Lee (2007) estudia a la industria del turismo en Corea del Sur aplicando ambos indicadores. Estas investigaciones evidencian la importancia del análisis del comportamiento económico en diferentes realidades.

De esta manera, se podría limitar el poder de los grandes grupos económicos existentes como es el caso de Ecuador, en donde hay explotación de la mano de obra contratada mediante salarios ínfimos que no igualan el salario 
mínimo vital, sin tener acceso a la canasta básica (Tulcanaza, 2010). En el marco de la inclusión, el gobierno debería poner en práctica políticas para fomentar e impulsar el emprendimiento, generando externalidades positivas, dentro de las cuales se encuentra el incremento del ingreso de las personas para emprender en negocios innovadores, la explotación de oportunidades, el incremento del empleo, la reducción de costos y el desarrollo tecnológico.

Albuja (2019) analiza la inclusión económica y social en Ecuador, sus resultados indican que para 2017, 67.2\% de la población se encuentra socialmente incluida, para lo cual se consideró a aquellas personas que no atraviesen condiciones de pobreza multidimensional; en tanto que $42.1 \%$ de ciudadanos se encuentran en circunstancias de inclusión productiva, es decir, aquellas personas con adecuado nivel de ingresos, empleo y seguridad social. El análisis concluye que $35.3 \%$ de la población se encuentra en un estado de doble inclusión.

La literatura muestra algunas herramientas para desarrollar la inclusión económica y social de todos los sectores, además de indicadores económicos para analizar la desigualdad, como son ingreso, renta, empleo, entre otros. Aunque también existe un instrumento adicional para incentivar la participación: el financiamiento, herramienta a desarrollar para lograr la inclusión de las MIPyME, ya que la disponibilidad limitada de financiamiento impulsa ciertos círculos viciosos que generan conflicto y bajo desarrollo, creando en las MIPyME una característica de fragilidad, lo que se vuelve un obstáculo en el crecimiento inclusivo. De esta forma, es obligatorio diseñar mecanismos para promover la inclusión financiera que permitan el acceso igualitario a los recursos económicos (Fowowe y Folarin, 2019).

Para contrarrestar la concentración económica y fomentar la inclusión empresarial, el fortalecimiento de los procesos de emprendimiento es tan importante como el acceso al financiamiento, puesto que las empresas nacientes enfrentan varios factores que determinan su competitividad y sostenibilidad, como son el nivel de educación de propietarios y administradores (Tambunan, 2019), su capacidad para percibir oportunidades de negocio en lugar de emprender por necesidad (Chairi et al., 2019), la gestión estratégica orientada al mercado que propicie la innovación y aprendizaje continuo (Tjandera y Hariandja, 2019), el acceso y uso oportuno de tecnología productiva y tecnología de información y comunicación (Farooq et al., 2020), y la disponibilidad de personal cualificado, productivo y eficiente (Guzmán-Soria et al., 2020).

La desigualdad económica es motivo de preocupación no sólo de entes gubernamentales sino de organismos mundiales. Así, el objetivo 8 de la Agenda 2030 propuesta por la Organización de las Naciones Unidas (ONU) plantea 
promover el crecimiento económico sostenido, inclusivo y sostenible, el empleo pleno y productivo y el trabajo decente para todos (Sustainable Development Goals Fund, 2019).

Para lograr el desarrollo sostenible propuesto por la onU, se sugiere que dentro de un país exista fortalecimiento de las instituciones con un mejoramiento en el marco normativo, y así poder mejorar el rendimiento de los recursos utilizados por las empresas. También, busca la convergencia entre países y mecanismos de integración para mejorar la vida de sus ciudadanos, citando como ejemplo lo logrado por la Unión Europea (Álvarez, 2016).

Trejo (2017) explica que las líneas fundamentales del desarrollo de las naciones son el crecimiento económico y la industrialización, tomando en cuenta el criterio de que se puede alcanzar una vida próspera y de calidad a través de una reestructuración económica e innovación, asignándole a todo esto un carácter inclusivo y sostenible.

Destaca la importancia de la equidad en cada uno de los sectores económicos como aporte a la equidad de la economía en su conjunto. Por tanto, se vuelve necesario evaluar la distribución de los ingresos por área de actividad económica, y buscar aquellos factores que permitan a las organizaciones una mayor participación en las mismas. El desempeño económico de las firmas constituye un predictor de la participación de éstas en la actividad económica y los ingresos del sector (Chiu, 1998; Lee et al., 1999). Un mejor desempeño económico permite una mayor competitividad, que se refleja en una mejor posición empresarial desde el punto de vista de ingresos (Jung et al., 2018). Por lo anterior, es fundamental determinar ciertos factores o variables sobre los que el gerente de la firma puede incidir para lograr un mejor desempeño y, por ende, una mejor posición competitiva.

\section{METOdología}

Con la finalidad de evaluar la concentración de ingresos e inclusión en el sector empresarial ecuatoriano, se estableció como unidad de análisis a las empresas formales que conforman los 21 sectores económicos del país para 2017. Se utilizó como fuente de información secundaria los estados financieros que las sociedades reportan anualmente a la Superintendencia de Compañías, Valores y Seguros del Ecuador. Las sociedades objeto de estudio se refieren a las empresas con personería jurídica que presentan su información financiera al ente de control, con responsabilidad de un profesional contable y el representante legal. 
Para determinar la concentración económica, se calculó para cada sector el coeficiente de Gini respecto a los ingresos y la utilidad neta. Esta metodología fue adaptada bajo la premisa de que el coeficiente de Gini es una medida robusta y confiable de desigualdad (Bandyopadhyay, 2017); por lo que es ampliamente utilizada para medir la concentración en la distribución de diferentes variables empresariales entre agentes económicos (Alonso, 2010; Dávila, 2004; Lee, 2007; Mathews y Schwartz, 2019; Mussini, 2019; Thomas y Kandaswamy, 2019; Zhao et al., 2007).

Después de establecer el nivel de concentración económica de los sectores, el análisis se enfocó en cuatro sectores prioritarios que representan el máximo y el mínimo nivel de concentración y los de mayor aporte al PIB del país.

Dada la importancia de análisis del desempeño económico, como factor determinante que contribuye a la participación de las empresas en una mejor posición competitiva dentro de los sectores económicos, es necesario definir variables para la medición del desempeño. Es de común uso como métrica de la gestión, el uso de indicadores financieros (ratios) (Block y Hirt, 2008), los cuales relacionan la información contable de las organizaciones y reflejan su quehacer empresarial. Para estudiar el comportamiento de la gestión financiera se llevó a cabo un análisis descriptivo y comparativo de indicadores financieros: rentabilidad sobre activos (ROA), rentabilidad sobre patrimonio (ROE), margen de utilidad neta (MUN), razón corriente, razón de endeudamiento, apalancamiento financiero, rotación de activos totales e intensidad de los activos; todos complementados con el análisis Du Pont y la antigüedad de las empresas. Las firmas se agruparon en dos unidades de análisis: la primera conformada por las empresas que pertenecen al decil de mayor concentración de ingresos, y la segunda compuesta por las que se sitúan bajo el noveno decil, con lo cual se evidencia el resultado de las medidas financieras de cada grupo.

Finalmente, para estimar la influencia de las razones financieras estudiadas sobre la probabilidad de que una empresa pertenezca al decil de mayor concentración de ingresos, se desarrolló un análisis de regresión logística (LOGIT), permitiendo visualizar un modelo que muestra las principales variables que intervienen en el comportamiento del sector desde el punto de vista de la concentración de ingresos.

Para estudiar la probabilidad de pertenecer al grupo de mayor concentración de ingresos, se estimó el siguiente modelo de regresión: 


$$
\begin{aligned}
\ln (1 / 0)= & \beta_{0}+\beta_{1} R O A+\beta_{2} E N D+\beta_{3} I A N \\
& +\beta_{4} A P F+\beta_{5} E d a d+u_{i}
\end{aligned}
$$

En donde $\ln (1 / 0)$ representa la probabilidad que tiene una empresa de formar parte del décimo decil de concentración de ingresos en su sector; la cual se explica por las siguientes variables: rentabilidad sobre activos (ROA), razón de endeudamiento (END), intensidad de activos netos (IAN), apalancamiento financiero (APF), edad expresada como el tiempo en ańos transcurrido desde la constitución de una firma hasta diciembre 2019 (edad).

\section{RESULTADOS Y DISCUSIÓN}

Una sociedad inequitativa, desde una perspectiva económica, es propensa a formar grupos sociales y económicos que defienden a grupos con mayor influencia, y generan resistencia al crecimiento de organizaciones que se encuentran en las menores escalas de la estructura distributiva; lo que conlleva a hablar de inclusión como un proceso a seguir en la búsqueda de una sociedad cohesiva.

La búsqueda de la adaptación permanente que presentan las empresas -por la globalización y el rápido avance tecnológico- ocasiona que las organizaciones enfrenten estos cambios en condiciones desiguales y segmenta a los sectores productivos. Es necesario, entonces, un análisis descriptivo del comportamiento de cada uno de los sectores empresariales en el afán de visualizar a los más representativos en la economía ecuatoriana, mismos que serán objeto de estudio y permitirán evaluar métricas pertinentes que evidencien su comportamiento y propicien el planteamiento de estrategias y políticas que faciliten la inclusión empresarial.

La tabla 1 presenta el número de empresas, ingresos y utilidad neta de los diferentes sectores económicos; se evidencia que los sectores de Manufactura (C) y Comercio $(\mathrm{G})$ fueron los que más aporte realizaron a la producción de bienes y servicios en Ecuador, toda vez que sus ingresos representan $62.4 \%$ del total generado por el sector empresarial ecuatoriano para 2017, así como $40.4 \%$ del total de las utilidades netas registradas.

La tabla 2 muestra el coeficiente de Gini por sector empresarial. Evidencia que todos los sectores económicos presentan desigualdad respecto a la obtención de ingresos y utilidad neta; es decir, un número reducido de empresas concentran la mayor cantidad de ingresos y beneficios en el país, mostrando 


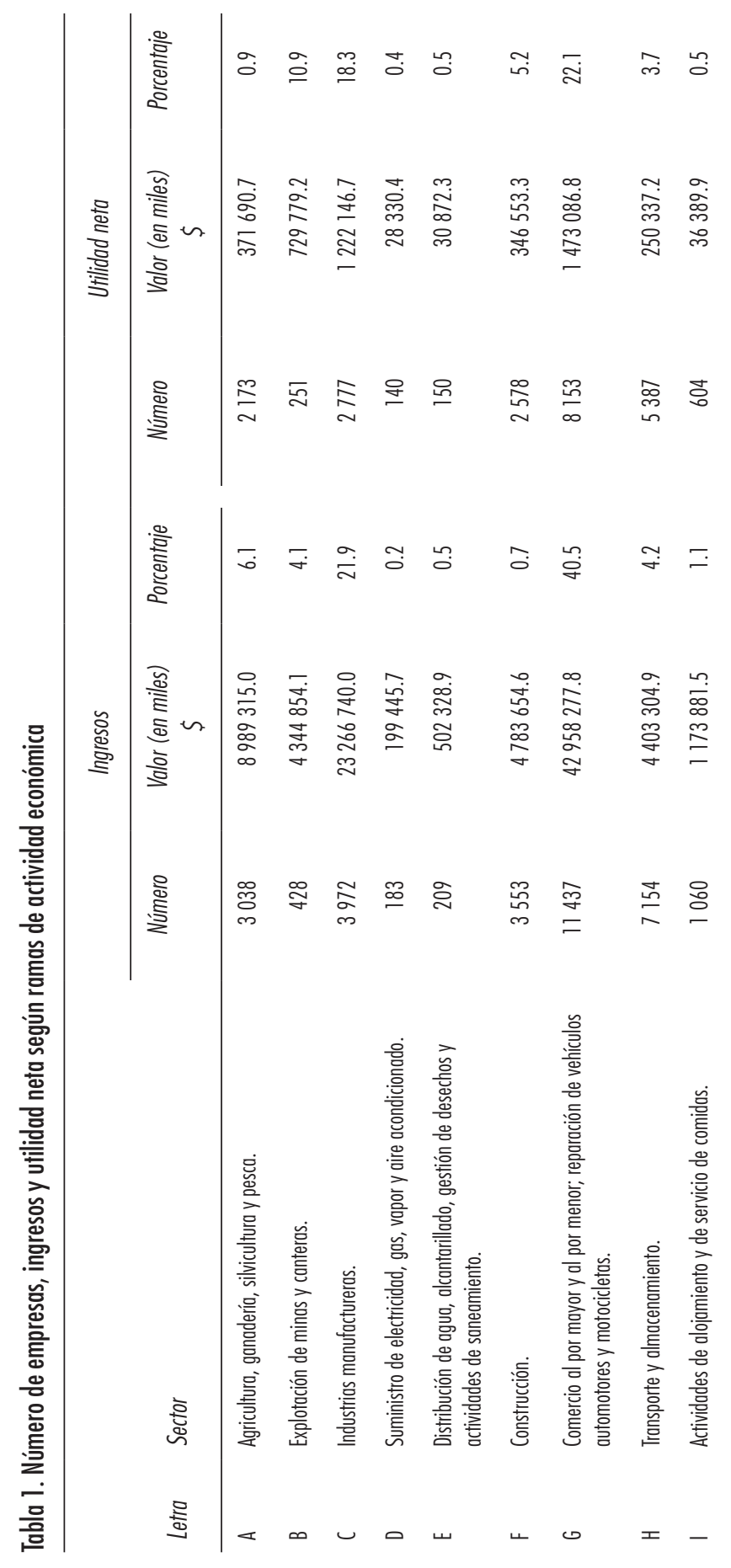




$$
\begin{aligned}
& \text { ๗ిల }
\end{aligned}
$$

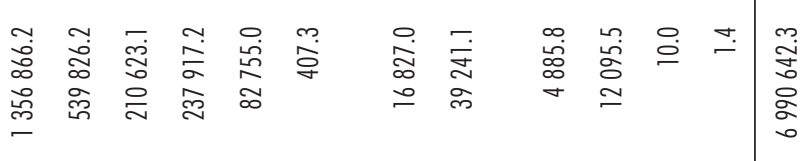

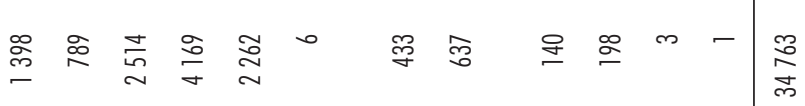

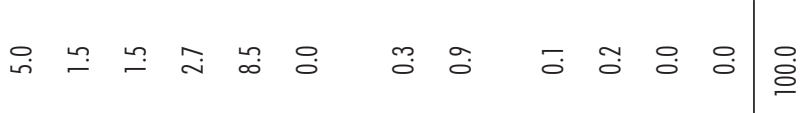

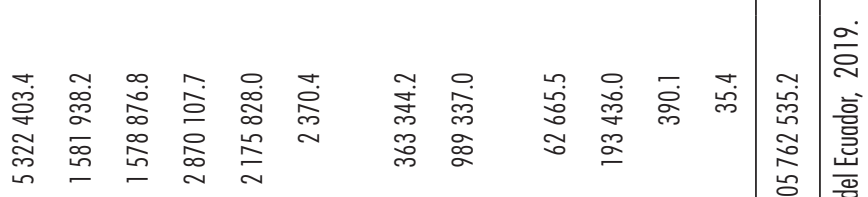

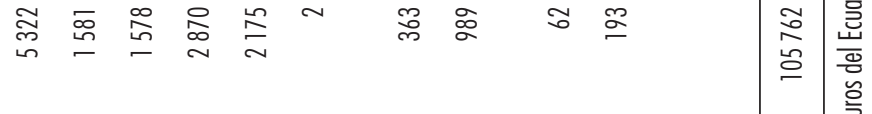

$$
\begin{aligned}
& \text { ๙̊) }
\end{aligned}
$$

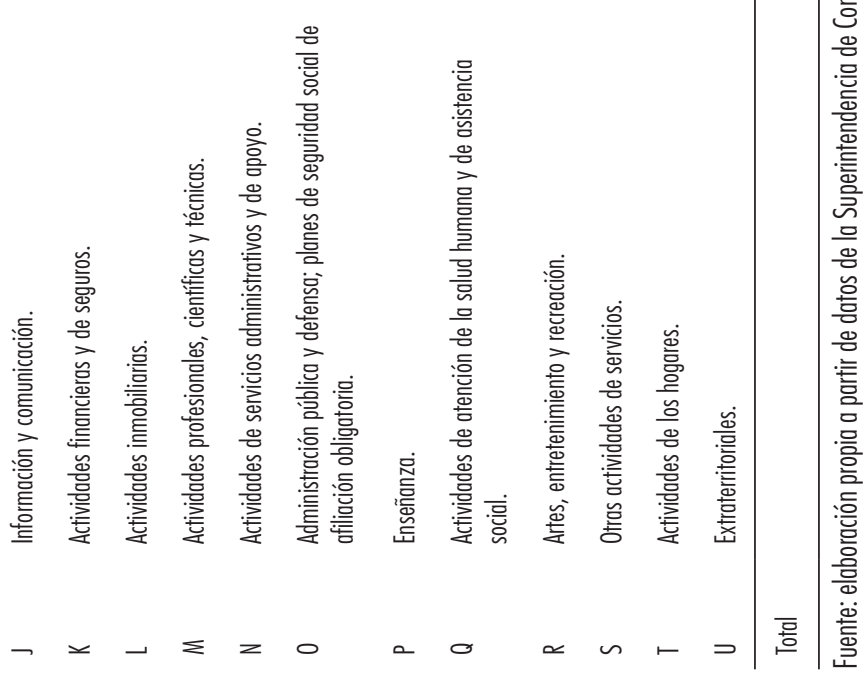


un desequilibrio importante en la distribución de recursos del sector empresarial. Situación que ratifica la concentración de riqueza y desigualdad para el caso de Ecuador, de forma análoga a lo expuesto por Tulcanaza (2010), Deere y Contreras (2011) y Solimano (2015); y por el Oxford Committee for Famine Relief (2018), respecto a la concentración de riqueza a nivel mundial. El sector que presenta mayor desigualdad, desde el punto de vista del ingreso, es el de Información y comunicación (J), en contraste con el sector Otras actividades de servicios (S) el cual presenta menor desigualdad.

Tabla 2. Índice de Gini según ramas de actividad económica

\begin{tabular}{llcc}
\hline Letra & Sector & $\begin{array}{c}\text { Gini } \\
\text { ingresos }\end{array}$ & $\begin{array}{c}\text { Gini } \\
\text { utilidad }\end{array}$ \\
\hline A & Agricultura, ganadería, silvicultura y pesca. & 0.78 & 0.81 \\
B & Explotación de minas y canteras. & 0.86 & 0.89 \\
C & Industrias manufactureras. & 0.84 & 0.87 \\
D & Suministro de electricidad, gas, vapor y aire acondicionado. & 0.76 & 0.86 \\
E & Distribución de agua, alcantarillado, gestión de desechos y actividades de saneamiento. & 0.83 & 0.86 \\
F & Construcción. & 0.83 & 0.87 \\
G & Comercio al por mayor y al por menor; reparación de vehículos automotores y motocicletas. & 0.83 & 0.86 \\
H & Transporte y almacenamiento. & 0.83 & 0.88 \\
I & Actividades de alojamiento y de servicio de comidas. & 0.77 & 0.82 \\
J & Información y comunicación. & 0.87 & 0.90 \\
K & Actividades financieras y de seguros. & 0.82 & 0.86 \\
L & Actividades inmobiliarias. & 0.81 & 0.85 \\
M & Actividades profesionales, cientíicas y técnicas. & 0.79 & 0.85 \\
N & Actividades de servicios administrativos y de apoyo. & 0.79 & 0.83 \\
P & Enseñanza. & 0.76 & 0.82 \\
Q & Actividades de atención de la salud humana y de asistencia social. & 0.78 & 0.79 \\
R & Artes, entretenimiento y recreación. & 0.73 \\
S & Otras actividades de servicios. & 0.85 \\
\hline
\end{tabular}

Fuente: elaboración propia a partir de datos de la Superintendencia de Compañías, Valores y Seguros del Ecuador, 2019. 
Los resultados obtenidos en el índice de Gini, si bien son basados en información financiera, evidencian la posible existencia de desequilibrios sociales como riesgos de inversión, empleo, emprendimiento, y permanencia en el sector.

Es importante el análisis financiero de los sectores más representativos de la economía, así como de aquellos que presentan los valores extremos en relación con el nivel de concentración. En la tabla 3 se muestran los principales indicadores que permiten visualizar el desempeño financiero de cada sector objeto de análisis.

Tabla 3. Estadísticos descriptivos de indicadores financieros

\begin{tabular}{lcccc}
\hline Indicadores & Manufactura (C) & Comercio (G) & $\begin{array}{c}\text { Información y } \\
\text { comunicación (J) }\end{array}$ & $\begin{array}{c}\text { Otras actividades } \\
\text { de servicio (S) }\end{array}$ \\
\cline { 2 - 5 } & Mediana & Mediana & Mediana & Mediana \\
\hline Rotación de activos totales & 1.252 & 1.499 & 1.643 & 1.491 \\
Apalancamiento financiero & 2.381 & 2.765 & 2.004 & 2.175 \\
Rentabilidad sobre activos & 0.020 & 0.017 & 0.025 & 0.021 \\
Rentabilidad sobre patrimonio & 0.081 & 0.087 & 0.123 & 0.098 \\
Margen de utilidad neta & 0.016 & 0.012 & 0.017 & 0.018 \\
Endeudamiento & 0.642 & 0.703 & 0.621 & 0.646 \\
Dupont & 0.081 & 0.087 & 0.123 & 0.098 \\
Razón corriente & 1.546 & 1.554 & 1.503 & 1.608 \\
Intensidad de activos netos & 0.221 & 0.070 & 0.055 & 0.137 \\
Edad & 10.000 & 9.000 & 6.000 & 10.000 \\
\hline
\end{tabular}

Fuente: elaboración propia a partir de datos de la Superintendencia de Compañías, Valores y Seguros del Ecuador, 2019.

En la figura 1 se presentan los datos del rendimiento sobre activos de los distintos sectores analizados por deciles con respecto al nivel de ingresos. Se puede observar que los sectores Manufacturera (C) y Comercio (G) poseen un comportamiento similar, demostrando que conforme crece el nivel de ingreso, el rendimiento sobre activos es ascendente. A diferencia, los sectores de Información y comunicación (J) y Otras actividades de servicios (S), los cuales son los extremos desde el punto de vista de nivel de concentración, tienen un comportamiento mucho más volátil, presentando picos y caídas pronunciadas al ser analizados por deciles. 
Figura 1. Rentabilidad sobre activos de los sectores estudiados por deciles en medianas

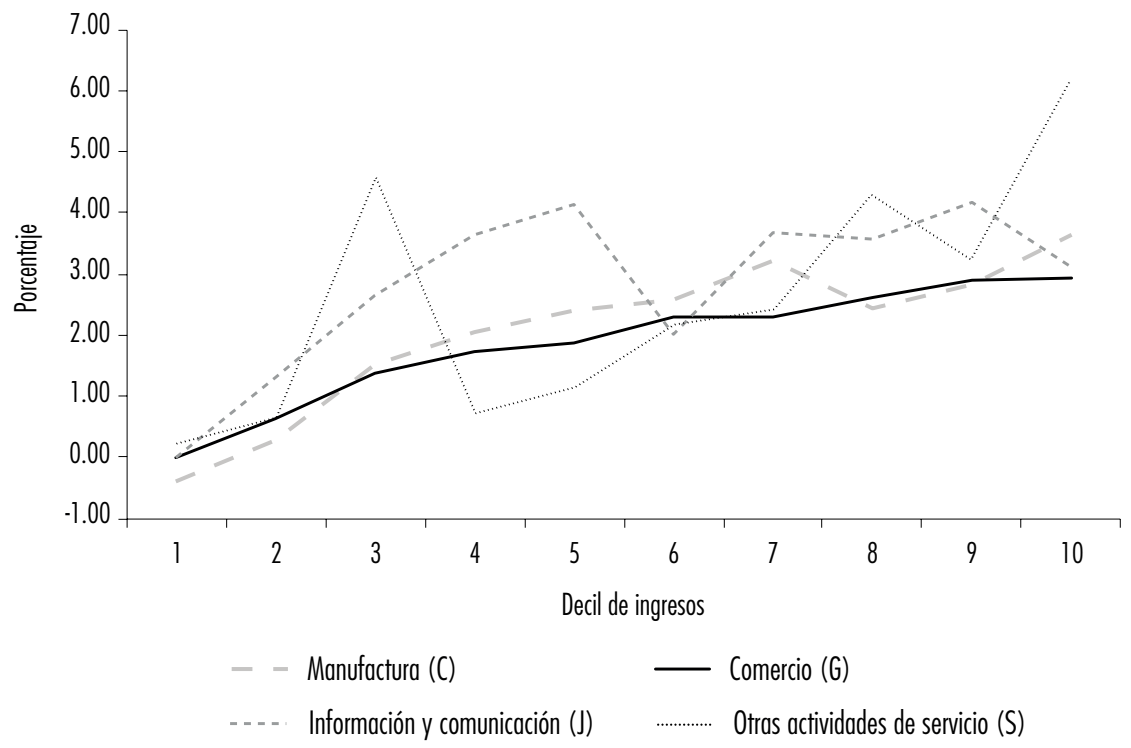

Fuente: elaboración propia a partir de datos de la Superintendencia de Compañías, Valores y Seguros del Ecuador, 2019.

La figura 2 indica que las empresas que se encuentran en los deciles de mayores ingresos muestran una intensidad de activos superior. El sector Manufactura $(\mathrm{C})$ presenta una tendencia de crecimiento en la intensidad de sus activos, llegando en su último decil a tener activos fijos 33 veces superiores al decil 1; por otro lado, el sector Otras actividades de servicios (S) presenta una volatilidad marcada que corresponde con las actividades diversas que realizan las empresas de este sector.

En la figura 3 se registra el apalancamiento financiero de las empresas de cada uno de los sectores estudiados; en donde se evidencia que a mayor nivel de ingresos las empresas optan por financiar sus actividades en mayor proporción con recursos de terceros o deuda. Tanto los sectores Manufactura (C) como Comercio $(\mathrm{G})$ tienen un comportamiento similar en el que la tendencia es creciente; es decir, financian sus actividades en su mayoría con recursos ajenos sobre financiamiento propio hasta alcanzar valores máximos en el decil 8 y 9, respectivamente; a partir de entonces, las empresas optan por financiar sus actividades con menor deuda y mayores recursos propios. El sector Información y comunicación $(\mathrm{J})$ tiene una sostenida tendencia positiva; por lo que las empresas con mayor cantidad de ingresos utilizan mayor cantidad de deuda. 
Figura 2. Intensidad de activos netos de los sectores estudiados por deciles en medianas

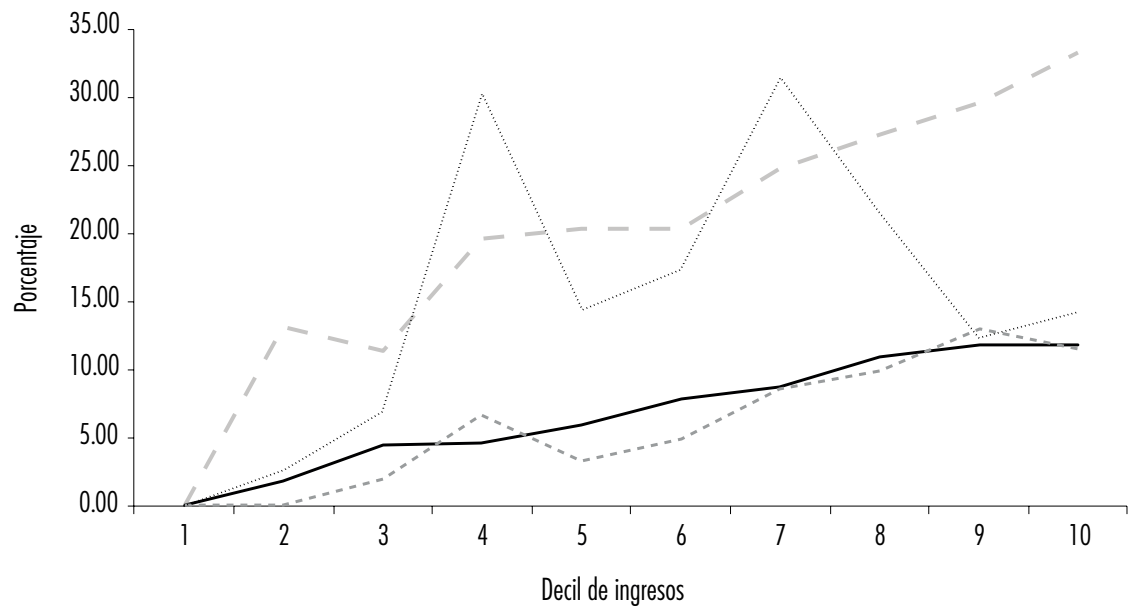

- - Manufactura (C)

- Comercio (G)

-.... Información y comunicación (J) Otras actividades de servicio (S)

Fuente: elaboración propia a partiri de datos de la Superintendencia de Compañías, Valores Seguros del Ecuador, 2019.

Figura 3. Apalancamiento financiero de los sectores estudiados por deciles en medianas

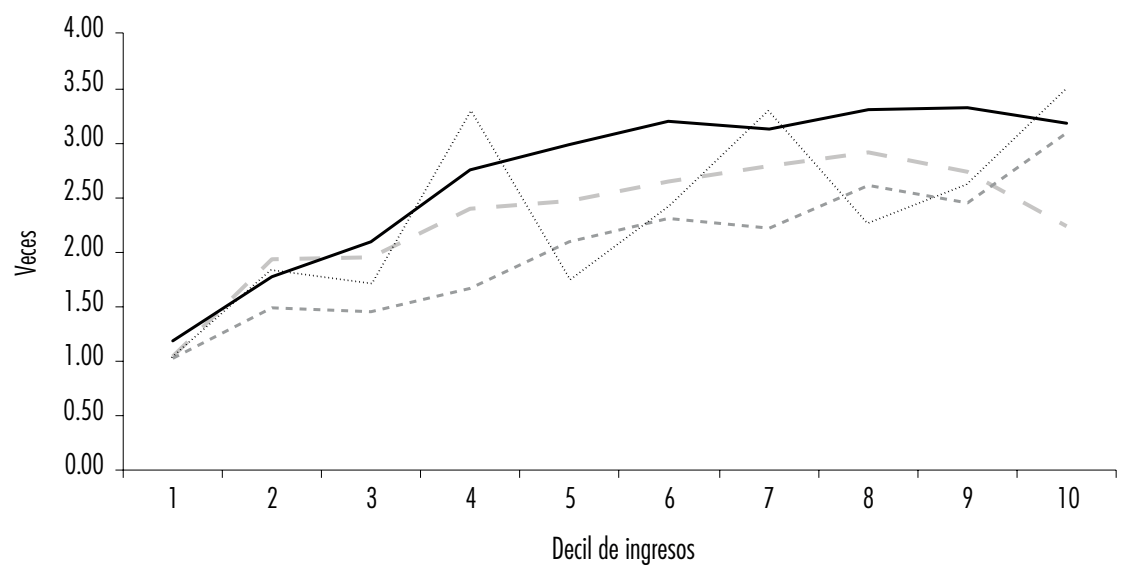

- - Manufactura (C)

- Comercio (G)

-.... Información y comunicación (J)

…….. Otras actividades de servicio (S)

Fuente: elaboración propia a partiri de datos de la Superintendencia de Compañías, Valores y Seguros del Ecuador, 2019. 
En la figura 4 se presenta la edad de las empresas en cada sector empresarial. Se evidencia en los cuatro sectores analizados que las empresas que concentran mayor cantidad de ingresos, en promedio, presentan mayor edad respecto a las que obtuvieron los menores ingresos. Los sectores Manufactura (C), Comercio $(\mathrm{G})$ e Información y comunicación $(\mathrm{J})$ presentan comportamientos similares, con una tendencia positiva que se acentúa en el último decil. El sector Otras actividades de servicios (S) que evidenció la menor desigualdad en ingresos, al igual que en indicadores anteriores, muestra un comportamiento volátil.

La figura 5 presenta el promedio de endeudamiento de cada sector de estudio. Se evidencia que, para todos los sectores, las empresas en los primeros deciles de ingresos presentan mayor endeudamiento, el cual disminuye conforme se incrementan los ingresos de las firmas.

En la tabla 4 se refleja el desempeño financiero de los sectores objeto de estudio. Se evidencia que, en promedio, el sector de Otras actividades de servicios (S) presenta mayores valores de razón corriente, intensidad de los activos netos y apalancamiento financiero; además, sus empresas en promedio presentan una mayor edad. Por lo anterior, los indicadores financieros estadísticamente significativos muestran indicios de una mejor gestión empresarial en el sector más equitativo.

Figura 4. Edad promedio de las empresas de cada sector estudiado por deciles

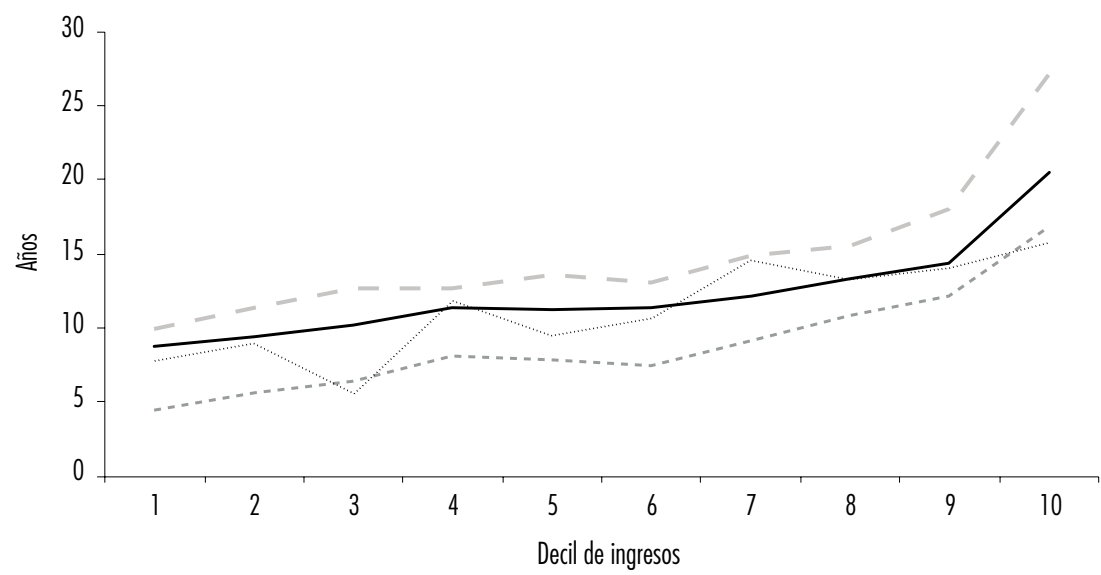

$$
\begin{aligned}
& \text { - - Manufactura (C) - Comercio (G) } \\
& \text { _..... Información y comunicación (J) _.......... Otras actividades de servicio (S) }
\end{aligned}
$$

Fuente: elaboración propia a partir de datos de la Superintendencia de Compañías, Valores y Seguros del Ecuador, 2019. 
Figura 5. Endeudamiento promedio de las empresas de cada sector estudiado por deciles

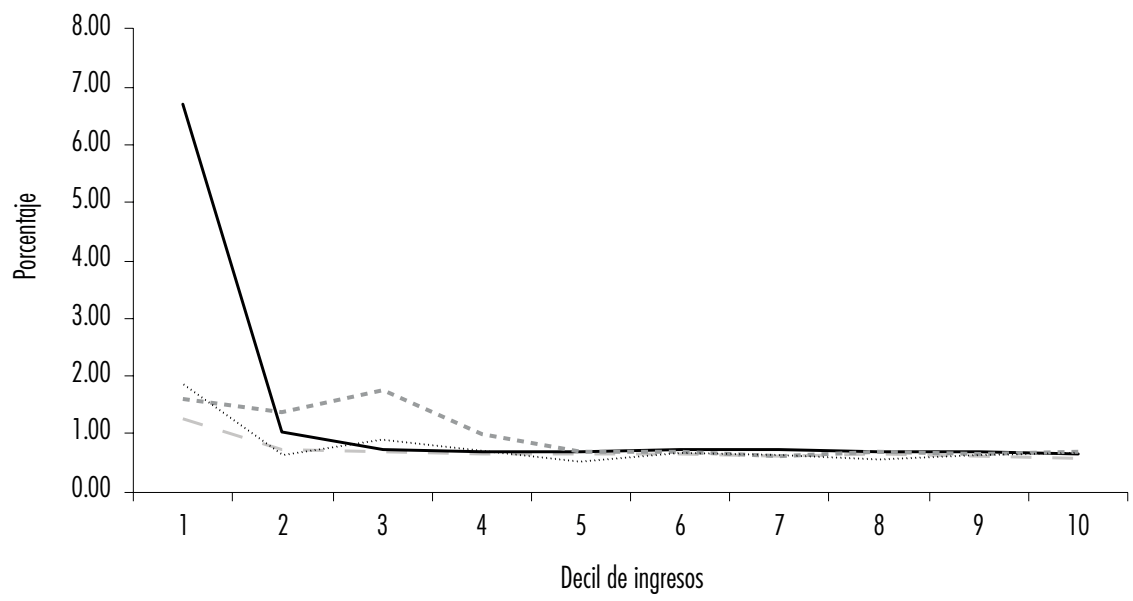

$$
\begin{aligned}
& \text { - }- \text { Manufactura (C) - Comercio (G) } \\
& \text {. . . . Información y comunicación (J) _........... Otras actividades de servicio (S) }
\end{aligned}
$$

Fuente: elaboración propia a partir de datos de la Superintendencia de Compañías, Valores y Seguros del Ecuador, 2019.

Tabla 4. Indicadores financieros promedio de los sectores con mayor y menor concentración

\begin{tabular}{lll}
\hline Sector & $\begin{array}{c}\text { Gini } 0.73 \\
\text { Otras actividades de servicios (S) }\end{array}$ & $\begin{array}{c}\text { Gini } 0.87 \\
\text { Información y comunicación (J) }\end{array}$ \\
\hline Rotación de activos totales & 2.19 & 2.25 \\
Apalancamiento financiero & $5.89^{\star *}$ & $4.09^{* *}$ \\
Rentabilidad sobre activos & 0.02 & 0.01 \\
Rentabilidad sobre patrimonio & 0.15 & 0.20 \\
Margen de utilidad neta & $-0.01^{\star *}$ & $-0.05^{* \star}$ \\
Endeudamiento & 0.60 & 0.63 \\
Dupont & 0.15 & 0.20 \\
Razón corriente & $4.11^{\star *}$ & $3.21^{* *}$ \\
Intensidad de activos netos & $0.24^{\star *}$ & $0.19^{* *}$ \\
Edad & $11.14^{\star *}$ & $8.90^{* *}$ \\
\hline
\end{tabular}

Nota: ** estadísticamente significativo al 0.05 para Test de Welch.

Fuente: elaboración propia a partir de datos de la Superintendencia de Compañías, Valores y Seguros del Ecuador, 2019. 
Para encontrar las diferencias estadísticamente significativas respecto al desempeño empresarial, entre las empresas que pertenecen al decil de mayor concentración de ingresos y aquellas que conforman los nueve deciles restantes, se llevó a cabo una prueba de medias para cada uno de los sectores económicos estudiados (véase tabla 5). Cabe destacar que en promedio las empresas que concentran la mayor cantidad de ingresos (grupo 1) presentan una mayor edad y rentabilidad sobre activos, así como menores niveles de razón corriente, situación que se presenta en todos los sectores de análisis.

En la tabla 6 se recogen resultados del modelo de regresión logística, el cual muestra la probabilidad de que una empresa se encuentre en el grupo de mayor concentración (1) o de menor concentración (0).

Los resultados evidencian para todos los sectores que, a mayor rentabilidad sobre activos, endeudamiento, y edad, la probabilidad de que una empresa forme parte del grupo de mayor concentración de ingresos se incrementa. En el caso de los sectores de Manufactura (C) y Comercio (G), que son los más representativos de la economía nacional, se muestra que el apalancamiento financiero es también una variable predictora que indica que a menores niveles de apalancamiento la probabilidad de pertenecer al grupo de mayor concentración aumenta.

El estudio demuestra que existe una amplia desigualdad en la generación de ingresos de las empresas en Ecuador, dando indicios de un inadecuado comportamiento que desemboca en un inequitativo crecimiento económico; por tanto, se vuelven imprescindibles ciertas políticas orientadas al desarrollo y apoyo de actividades operativas mediante la aplicación de metodologías de promoción de la productividad, que conlleven de manera implícita la promoción del trabajo justo y formal, el emprendimiento, y la innovación. La implementación de sistemas de control interno que reduzcan la probabilidad de comportamientos empresariales que vulneren las regulaciones antimonopolio, la asociación estratégica entre la academia y las MIPyME que posibilite a estas organizaciones acceder a innovación y tecnología que incremente su productividad y competitividad, el correcto direccionamiento de incentivos tributarios y el acceso efectivo a fondos de capital semilla, son posibles medidas de política industrial encaminadas a revertir la concentración económica.

Asimismo, el estudio evidencia en sus primeros deciles los mayores grados de desigualdad; por lo que se vuelve necesario el planteamiento de políticas que fomenten la formalización de las MIPyME, en donde puedan formar parte de un mercado competitivo justo y un sistema financiero nacional que fomente el acceso a recursos económicos, y permita su permanencia en el sec- 


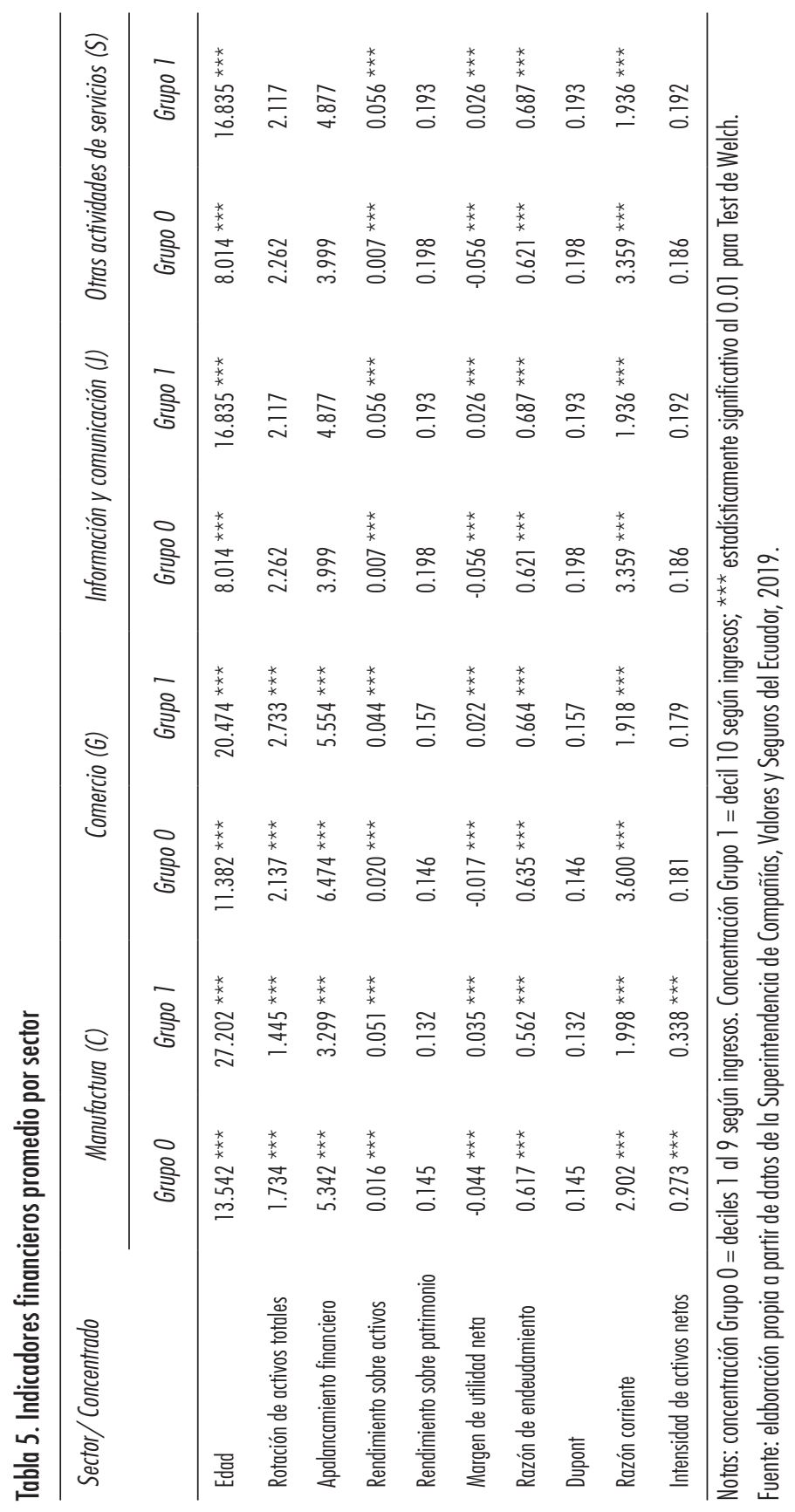




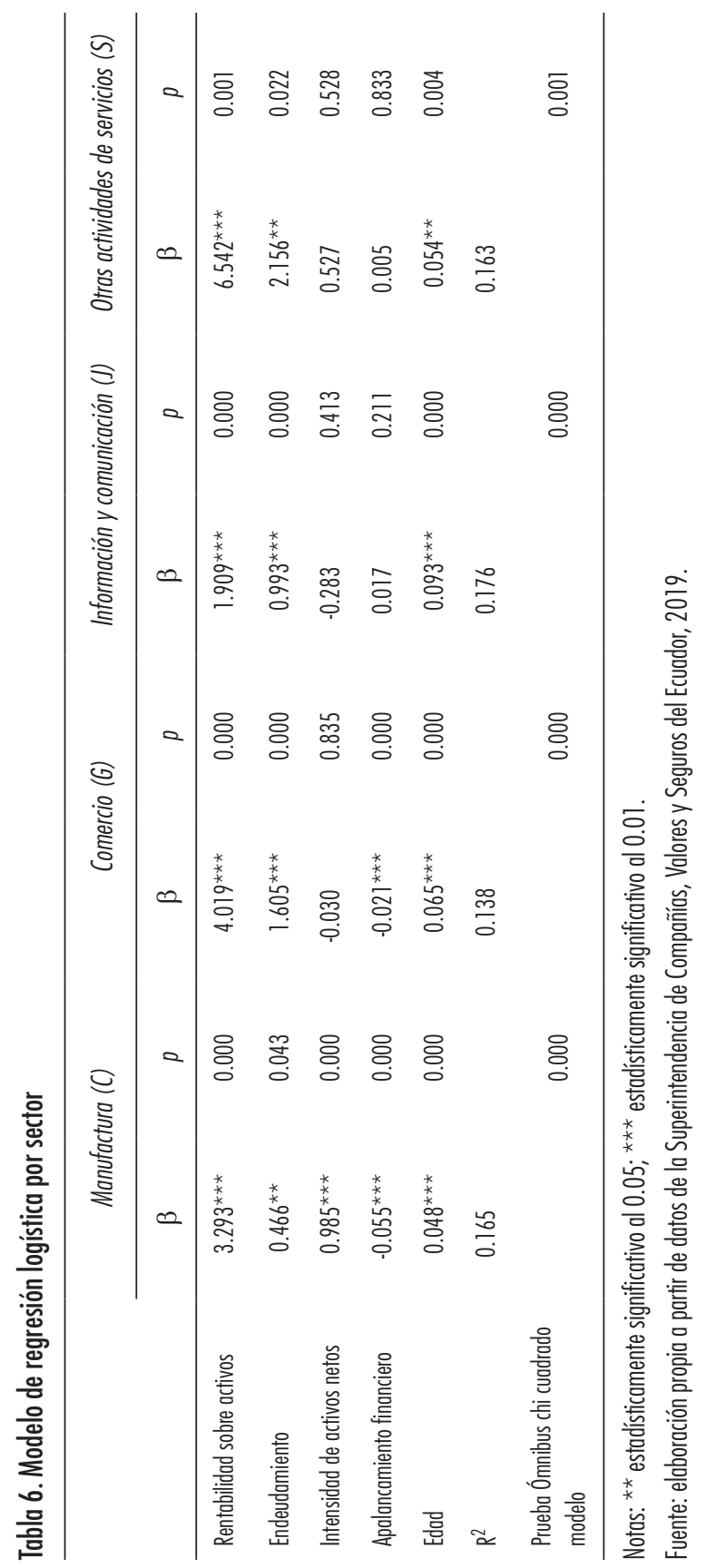


tor, necesidades que se destacan también en los estudios de Infante y Sunkel (2009), Abosede y Onakoya (2013), Fowowe y Folarin (2019) y Apetrei et al. (2019).

Desde el sector empresarial son necesarias ciertas acciones guiadas por los directivos, que vayan encaminadas a una inserción adecuada en el sector, con acciones que garanticen un crecimiento económico sostenido, inclusivo y sustentable, mediante estrategias dirigidas a un apropiado asesoramiento técnico sobre el manejo económico y productivo, encaminando los objetivos empresariales hacia las políticas nacionales y sectoriales, garantizando adecuadas condiciones internas de trabajo, y el apoyo al recurso humano que impacta en un mayor bienestar social dentro del sector.

\section{CONCLUSIONES}

Un coeficiente de Gini cercano a uno muestra un sector empresarial altamente desigual, lo que se asocia a un país con poca solidez económica y con un deficiente Estado de bienestar; en el que, la fuerza de las élites empresariales domina el entorno económico.

El estudio demostró una alta concentración de ingresos y beneficios en todos los sectores de la economía ecuatoriana, puesto que los índices de Gini superan el 0.7; siendo el sector de Información y comunicación (J) el que presenta mayor desigualdad y el sector Otras actividades de servicios (S) el menos concentrado.

El sector Otras actividades de servicios ( $\mathrm{S}$ ), que es considerado el más equitativo dentro de la economía, muestra volatilidad en todos los indicadores financieros analizados; es decir, su comportamiento es muy variable conforme se incrementa su decil de ingresos. Los sectores Manufactura (C) y Comercio $(\mathrm{G})$, que son los más representativos, muestran en los indicadores rentabilidad sobre activos, intensidad de activos netos, apalancamiento financiero, y edad, un comportamiento homogéneo, y además creciente, lo que evidencia que a mayor nivel de ingresos mayores son las variables mencionadas.

La comparación entre los sectores de mayor y menor concentración dio como resultado que las variables razón corriente, intensidad de activos netos, apalancamiento financiero y edad tuvieran diferencias estadísticamente significativas. Es importante destacar que las empresas del sector con menor concentración presentan mayores niveles de liquidez y de apalancamiento financiero. 
En el modelo de regresión logística se pudo apreciar que las variables rentabilidad sobre activos, endeudamiento y edad, influyen de manera positiva en la probabilidad de pasar a formar parte del grupo éxito (1) o de mayor concentración. Cabe destacar que las variables mencionadas son predictoras independientes del nivel de concentración de ingresos del sector. Adicionalmente, se pudo determinar, que el apalancamiento financiero influye de manera negativa en los sectores que concentran la mayor generación económica del país.

Los resultados del estudio evidencian la falta de autorregulación del mercado, y la necesidad de intervención del Estado, como agente externo, para establecer políticas y normas que promuevan la distribución equitativa de recursos.

Las políticas empresariales alineadas a las políticas públicas, encaminadas a desarrollar empresas sostenibles, no sólo deben centrar sus esfuerzos en una inclusión económica, sino en empresas con un enfoque de internacionalización que se desarrollen en un contexto de responsabilidad social empresarial, promoviendo un equilibrio entre crecimiento económico, bienestar social y aprovechamiento de recursos naturales y medio ambiente.

El debate sobre el crecimiento económico justo y equitativo es un tema que debe ser de análisis permanente, sobre todo, en países en vías de desarrollo. El presente análisis muestra una aproximación a la realidad del comportamiento económico y a la acumulación existente de riqueza en pequeños grupos, en diferentes sectores empresariales, aunque siempre será necesario el diagnóstico periódico del ambiente empresarial que posibilite el planteamiento de estrategias y políticas que propicien la inserción de grupos económicos rezagados.

\section{BIBLIOGRAFÍA}

Abosede, A. J. y Onakoya, A. B. (2013). Entrepreneurship, economic development, and inclusive growth. International Journal of Arts and Entrepreneurship, 1(3). https://publication.babcock.edu.ng/asset/docs/publications /ECON/9504/2070.pdf

Albuja, W. (2019). Inclusión productiva y social en Ecuador. Problemas del Desarrollo. Revista Latinoamericana de Economía, 50(197). https://doi. org/10.22201/iiec.20078951e.2019.197.64747

Alonso, O. (2010). Measuring concentration: Lorenz curves and their decompositions. Annals of Regional Science, 47(1). https://doi.org/10.1007/ s00168-010-0369-5 
Álvarez, A. M. (2016). Retos de América Latina: Agenda para el Desarrollo Sostenible y Negociaciones del siglo xxi. Problemas del Desarrollo. Revista Latinoamericana de Economia, 47(186). http://dx.doi.org/10.22201/ iiec.20078951e.2016.186.55886

Apetrei, A., Sánchez, J. L. y Sapena, J. (2019). The controversial link between entrepreneurial activity and inequality. International Entrepreneurship and Management Journal, 15(1). https://doi.org/10.1007/s11365-019-00 $560-1$

Arbeletche, P. y Carballo, C. (2008). La expansión agrícola en Uruguay: algunas de sus principales consecuencias. Revista de Desarrollo Ruraly Cooperativismo Agrario, 12(7).

Banco Mundial (2000). Informe sobre el Desarrollo Mundial. http://documents 1.worldbank.org/curated/en/509031468137396214/pdf/2268 40SPANISH0WDR0200002001.pdf

Ballet, J., Bazin, D. y Mahieu, F. (2020). A policy framework for social sustainability: Social cohesion, equity and safety. Sustainable Development, 28(5). https://doi.org/10.1002/sd.2092

Bandyopadhyay, S. (2017). The absolute Gini is a more reliable measure of inequality for time dependent analyses (compared with the relative Gini). Economics Letters, 162(1). https://doi.org/10.1016/j.econlet.2017.07.012 Block, S. y Hirt, G. (2008). Fundamentos de administración financiera. McGraw Hill.

Calvo, R., Portet, J. y Bou, M. (2016). La equidad social como elemento esencial para el desarrollo local. En J. Márquez, J. Jurado y F. Pazos (coord.). Desarrollo local en territorios de fronteras (pp. 1-11). Servicio de Publicaciones Universidad de Huelva.

Carneiro, J., Matos, N. y Husted, B. (2015). Free markets and social inclusion: Toward a common goal. Journal of Business Research, 68(2). https:// doi.org/10.1016/j.jbusres.2014.09.025

Chairi, Z., Afrita, A. y Yudhistira, E. (2019). Venture capital as an alternative funding for micro small and medium enterprises (MSME) in Medan. Journal Jurisprudence, 9(2). https://doi.org/10.23917/jjr.v9i2.9020

Chiu, W. H. (1998). Income inequality, human capital accumulation and economic performance. The Economic Journal, 108(3). https://www.jstor. org/stable/2565736\%0A

Dávila, A. (2004). México: concentración y localización del empleo manufacturero, 1980-1998. Economía Mexicana. Nueva Época, 13(2). https:// www.redalyc.org/articulo.oa?id=323/32313204 
Deere, C. y Contreras, J. (2011). Acumulación de activos. Una apuesta por la equidad. Flacso, Sede Ecuador.

Farooq, R., Hassan, G., Padhy, N., Peerzad, S. A. e Ismail, A. (2020). The utilization of Information and Communication Technology (ICT). In the MSME's of the J\&K, with its impacts. 2020 International Conference on Computer Science, Engineering and Applications (ICCSEA), 1(6). https:// doi.org/10.1109/ICCSEA49143.2020.9132953

Fowowe, B. y Folarin, E. O. (2019). The effects of fragility and financial inequality inclusive growth on African countries. Review of Development Economics, 36(23). https://doi.org/10.111 1/rode.12594

Guzmán-Soria, E., Garza-Carranza, M. T., García-Salazar, J. A., HernándezMartínez, J. y Rebollar-Rebollar, S. (2020). Productivity and efficiency analysis of msmes in Mexico. Journal Globalization, Competitiveness and Governability, 14(2). https://doi.org/10.3232/GCG.2020.V14.N2.03

Husted, B. W. (2011). Social equity as a concern of management: the role of income inequality. Academy of Management Proceedings, 2011(1). https:// doi.org/10.5465/ambpp.2011.65870527

Infante, R. y Sunkel, O. (2009). Chile: hacia un desarrollo inclusivo. Revista de la CEPAL, 97(7). https://doi.org/10.18356/a30a6295-es

Jung, H., Seo, I. y Jung, K. (2018). Mediating role of entrepreneurship in explaining the association between income inequality and regional economic performance. Economic Development Quarterly, 32(2). https://doi. org/10.1177/0891242418758438

Lee, J., Lucius, H. y McNeil, S. (1999). Entrepreneurial rewards and economic performance: An empirical study of the best small companies. International Journal of Technology Management, 17(7). https://doi.org/https:// doi.org/10.1504/IJTM.1999.002748

Lee, C-K. (2007). Input-output analysis and income distribution patterns of the tourism industry in South Korea. Asia Pacific Journal of Tourism Research, 35(1). https://doi.org/10.1080 / 10941669708721962

Mathews, T. y Schwartz, J. A. (2019). Comparisons of utility inequality and income inequality. Economics Letters, 178(18). https://doi.org/10.1016/j. econlet.2019.02.015

Mussini, M. (2019). A spatial decomposition of the shift-share components of labor productivity inequality in Italy. Papers in Regional Science, 98(1). https://doi.org/10.1111/pirs.12362

Ncube, M., Soonawalla, K. y Hausken, K. (2019). The links between business environment, economic growth and social equity: A study of African 
countries. Journal of African Business, O0(00). https://doi.org/10.1080/15 228916.2019.1695184

Nguyen, H. N., Le, Q. H. y Nguyen, T. T. (2020). The linkages between growth, poverty, and inequality in Vietnam: An empirical analysis. Accounting, 6(2). http://dx.doi.org/10.5267/j.ac.2019.10.005

Organización de las Naciones Unidas para la Educación, la Ciencia y la Cultura (unesco) (1989). Convención sobre los Derechos del Niño. unesCo. Organización Internacional del Trabajo (оIт) (2017). El empleo productivo y el trabajo decente como fuente de prosperidad en América Latina y el Caribe. Oficina Regional de la OIT para América Latina y el Caribe.

Oxford Committee for Famine Relief (2018). Informe Anual de Latinoamérica y el Caribe. Oxford Committee for Famine Relief. https://oxfami library.openrepository.com/bitstream/handle/10546/620721/bp-hittingthe-target-aid-inequality-080419-es.pdf

Solimano, A. (2015). Crecimiento, desigualdad y democracia: la transformación capitalista en Chile. Centro Internacional de Globalización y Desarrollo.

Superintendencia de Compañías, Valores y Seguros del Ecuador (2019). Portal de información. https://appscvsmovil.supercias.gob.ec/portalInformacion/sector_societario.zul

Sustainable Development Goals Fund (2019). Objetivos de Desarrollo Sostenible, Objetivo 8: Trabajo decente y crecimiento económico. https://www.sdgfund.org/es/objetivo-8-trabajo-decente-y-crecimientoecon\%C3\%B3mico

Tambunan, T. (2019). Recent evidence of the development of micro, small and medium enterprises in Indonesia. Journal of Global Entrepreneurship Research, 9(1). https://doi.org/10.1186/s40497-018-0140-4

Thomas, D. y Kandaswamy, A. (2019). Identifying high resource consumption areas of assembly-centric manufacturing in the United States. The Journal of Technology Transfer, 44(1). https://doi.org/10.1007/s10961017-9577-9

Tjandera, W. y Hariandja, E. S. (2019). Linking organizational learning, organizational culture, and market orientation on innovation culture: A case study in Indonesian MSME. International Conference on Industrial Engineering and Operations Management, 23(7). https://bit.ly/38WDzq8

Trejo, A. (2017). Crecimiento económico e industrialización en la Agenda 2030: perspectivas para México. Problemas del Desarrollo. Revista Latinoamericana de Economia, 48(188). https://doi.org/10.1016/j.rpd.2017.01.005 
Trudeau, D. (2018). Integrating social equity in sustainable development practice: Institutional commitments and patient capital. Sustainable Cities and Society, 41. https://doi.org/10.1016/j.scs.2018.05.007

Tulcanaza, A. B. (2010). Concentración del poder económico en el sector comercio en Ecuador, periodo 2002-2008. Pontificia Universidad Católica del Ecuador.

Valbuena, P. y Montenegro, Y. (2019). Los negocios internacionales y el relacionamiento económico: una revisión de la literatura. Revista Facultad de Ciencias Económicas, 27(1). https://doi.org/10.18359/rfce.3233

Zhao, L., Sun, L., Yang, H. y Li, B. (2007). Regional disparities in China's manufacturing industry from 1987 to 2003. 2007 International Conference on Management Science and Engineering. https://doi.org/10.1109/ ICMSE.2007.4422009 